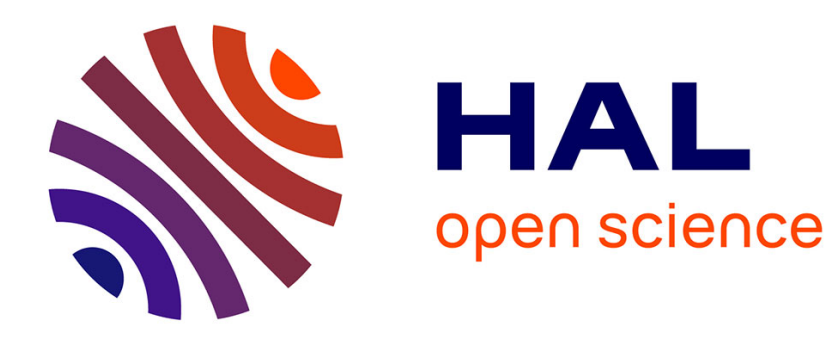

\title{
Possibilité de déconvolution du signal vidéo
}

\author{
E. Vicario, M. Pitaval
}

\section{To cite this version:}

E. Vicario, M. Pitaval. Possibilité de déconvolution du signal vidéo. Revue de Physique Appliquée, 1974, 9 (2), pp.389-391. 10.1051/rphysap:0197400902038900 . jpa-00243792

\section{HAL Id: jpa-00243792 https://hal.science/jpa-00243792}

Submitted on 1 Jan 1974

HAL is a multi-disciplinary open access archive for the deposit and dissemination of scientific research documents, whether they are published or not. The documents may come from teaching and research institutions in France or abroad, or from public or private research centers.
L'archive ouverte pluridisciplinaire HAL, est destinée au dépôt et à la diffusion de documents scientifiques de niveau recherche, publiés ou non, émanant des établissements d'enseignement et de recherche français ou étrangers, des laboratoires publics ou privés. 


\title{
POSSIBILITÉ DE DÉCONVOLUTION DU SIGNAL VIDÉO
}

\author{
E. VICARIO et M. PITAVAL \\ Département de Physique des Matériaux \\ Université Claude-Bernard, Lyon I, France
}

Résumé. - Le signal vidéo $s(t)$ d'un M. E. B. est en fait un produit de convolution.

$$
\dot{e}(t) * f(t)=\int_{0}^{t} e(x) f(t-x) \mathrm{d} x
$$

où $f(x)$ est la répartition spatiale des électrons émis pour chaque point de l'échantillon; $e(t)$ serait le signal obtenu si la zone émissive était infiniment petite. En prenant pour $f(x)$ une somme pondérée de deux gaussiennes pour représenter respectivement les électrons rétrodiffusés et les secondaires vrais, il est possible de résoudre l'équation de déconvolution :

$$
e(t)=s(t) \underset{*}{*} f(x)
$$

et d'obtenir ainsi une meilleure résolution. Pour l'instant, on se contente de traiter une seule ligne de balayage : le signal $s(t)$ est échantillonné, la courbe est lissée par une combinaison linéaire de polynômes de Tchebytcheff et la courbe résultante $e(t)$ est également représentée par des polynômes de Tchebytcheff.

En optimisant la fonction $f(x)$, on peut espérer obtenir des renseignements quantitatifs sur l'émission secondaire : répartition spatiale des rétrodiffusés et des secondaires et rapport des intensités émises.

Abstract. - The video signal $s(t)$ from a S. E. M. is in reality the convolution product.

$$
e(t) * f(t)=\int_{0}^{t} e(x) f(t-x) \mathrm{d} x
$$

where $f(x)$ is the spatial distribution of emitted electrons (secondaries and/or backscattered) at each point of the sample; $e(t)$ would be the expected signal if the electron probe and emitting zone were of infinitesimal dimensions. Assuming $f(x)$ is a gaussian repartition of backscattered electrons or a weighted sum of two gaussian functions when the signal of secondaries is used, it is possible to solve the deconvolution equation

$$
e(t)=s(t) \underset{*}{*} f(t)
$$

and a better resolution is then obtainable. At this time, only one slow scan line is treated : the signal $s(t)$ is sampled, the curve is fitted by a linear combination of Tchebytcheff polynomials, and the resulting curve $e(t)$ is represented in the form of Tchebytcheff polynomials.

Optimizing the function $f(x)$ it seems possible to obtain some information on the emission : spatial distribution of backscattered electrons, spatial distribution of secondaries and ratio of intensities between secondaries and backscattered electrons.

1. Introduction. - La résolution du microscope à balayage est limitée parfois par la taille du spot sur l'échantillon, presque toujours par des phénomènes physiques à l'intérieur de l'échantillon lui-même : élargissement du faisceau primaire dans le matériau, diffusion des porteurs dans le cas d'un semiconducteur.

Dans tous les cas, pour une position donnée du faisceau primaire, la zone émissive est une surface finie ; ou bien encore, en courant induit, la zone perturbée est un volume fini. Or, les électrons ou les photons détectés à ce moment-là fournissent un signal dont l'amplitude est affectée à un point de l'écran, c'est-à-dire à une surface supposée infiniment petite. On aurait donc une image " parfaite » si l'on pouvait à un instant donné ne recueillir que les électrons provenant d'un point de l'échantillon.

2. Le signal vidéo est un produit de convolution. Nous désignons par $e(x)$ un tel signal. Si le faisceau primaire est fixe, $e(x)$ est une impulsion de Dirac $\delta\left(x_{0}\right)$. En fait puisque la surface émissive est finie, on obtient, en réponse à cette impulsion, une répartition spatiale de l'émission $h\left(x-x_{0}\right)$. Si maintenant le faisceau primaire balaye l'échantillon, dans la direc- 

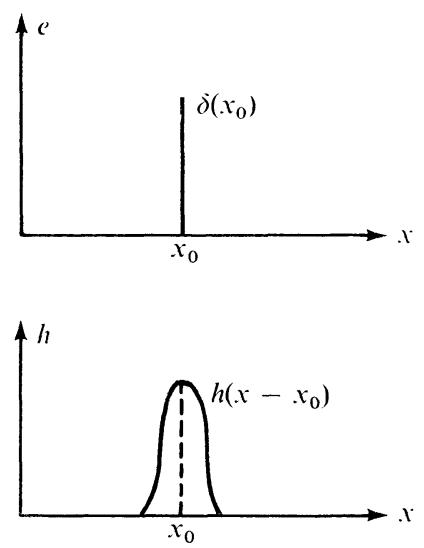

tion $x$, avec une vitesse uniforme, le signal vidéo $s(t)$ sera le produit de convolution des fonctions $e$ et $h$

$$
s(t)=\int_{0}^{t} e(\tau) h(t-\tau) \mathrm{d} \tau,
$$

soit symboliquement

$$
s(t)=e(t) * h(t)
$$

La fonction $h$ étant bornée et symétrique (1) s'écrit :

$$
s(t)=\int_{-\alpha}^{\alpha} e(t-y) h(y) \mathrm{d} y
$$

( $\pm \alpha$ étant les bornes de $h$ ).

A partir de $s(t)$, supposant connu $h(t)$, on peut espérer calculer

$$
e(t)=s(t) \stackrel{*}{*} h(t) .
$$

Malheureusement $s(t)$ est entachée de bruit $b(t)$ et $h(t)$ s'accompagne d'une incertitude $i(t)$ et, donc, on cherchera à résoudre

$$
e(t)=[s(t)+b(t)]_{*}^{*}[h(t)+i(t)]
$$

opération qui, en général, n’est pas réalisable ! [1].

3. Première approche de la solution. - On a cherché à résoudre cette équation pour une ligne seulement grâce à un ordinateur. Le signal $s(t)$ doit donc d'abord être échantillonné :

- soit en temps réel sur le signal vidéo lui-même,

- soit sur une courbe enregistrée ou photographiée,

- soit sur un négatif photographique.

La résolution se fait alors en 3 étapes :

$3.11^{\text {re }}$ ÉTAPE : LISSAGE. - La courbe $s(t)$ est approximée par une combinaison linéaire de polynômes de Tchebycheff

où

$$
s(t)=\sum_{k=0}^{k=n} a_{k} T_{k}
$$

$$
\mathrm{T}_{k}=\cos (k \operatorname{Arc} \cos t)
$$

avec $-1 \leqslant t \leqslant 1$.
Les coefficients $a_{k}$ sont calculés en minimisant la somme des carrés des écarts entre la courbe lissée et les points expérimentaux (méthode classique des moindres carrés). La difficulté réside dans le choix du degré maximum optimal $n_{0}$ des polynômes. Pour des valeurs de $n$ inférieures à $n_{0}$ la courbe sera mal lissée ; pour des valeurs supérieures à $n_{0}$ la courbe lissée s'entache de bruit. Des essais préliminaires permettent aisément de choisir ; mais il est difficile d'automatiser ce choix.

Lorsque le bruit est négligeable, le lissage n'a pour but que le choix de $n_{0}$ : on peut en effet penser raisonnablement que la courbe $e(t)$ sera bien représentée par une combinaison linéaire de polynômes de Tchebycheff de degré maximum $n_{0}$. Si le bruit est trop important, on pourra effectuer la déconvolution à partir de la courbe lissée.

$3.22^{\mathrm{e}}$ ÉTAPE : DÉCONVOLUTION. - $e(t)$ étant de la forme

$$
\sum_{k=0}^{k=n_{0}} a_{k}^{\prime} T_{k}(t)
$$

l'éq. (2) s'écrit

$$
\begin{aligned}
s(t) & =\int_{-\alpha}^{\alpha} \sum_{k} a_{k}^{\prime} T_{k}(t-y) h(y) \mathrm{d} y \\
& =\sum_{k} a_{k}^{\prime} \int_{-\alpha}^{\alpha} T_{k}(t-y) h(y) \mathrm{d} y .
\end{aligned}
$$

On calcule donc les intégrales

$$
\int_{-\alpha}^{\alpha} T_{k}(t-y) h(y) \mathrm{d} y
$$

et les coefficients $a_{k}^{\prime}$ sont encore obtenus par la méthode des moindres carrés.

$3.33^{\text {e }}$ ÉTAPE : OPTIMISATION. - La fonction $h(t)$ n'est pas ici une «fonction d'appareillage » comme dans un problème classique de déconvolution. C'est une "fonction d'échantillon " qui, donc, varie d'un matériau à l'autre et qui dépend du mode de fonctionnement du microscope, de l'énergie des électrons primaires, etc... Il ne semble pas d'ailleurs qu'on puisse toujours l'obtenir expérimentalement: par exemple, on peut mesurer le rapport entre le nombre d'électrons secondaires et le nombre de rétrodiffusés détectés ; mais ce n'est pas ce rapport qui intervient dans la fonction $h$ car de nombreux électrons secondaires ont été créés par des rétrodiffusés, soit dans l'échantillon lui-même, soit dans son environnement ; de tels secondaires portent l'information des rétrodiffusés et doivent être comptés comme tels. La fonction $h$ doit donc être choisie d'après des considérations plus ou moins théoriques ; on l'exprimera en fonction d'un certain nombre de paramètres et, en faisant varier ces derniers, on cherchera à minimiser la somme des carrés des écarts. Par exemple, dans le cas de l'émission secondaire, la fonction $h$ pourra être la somme pondérée de deux gaussiennes, l'une représentant les secondaires 
vrais et l'autre les rétrodiffusés ; on devra donc optimiser 3 paramètres: l'écart type de chacune des gaussiennes et leur pondération relative.

On entrevoit alors la possibilité d'atteindre ainsi certaines grandeurs physiques inaccessibles à la mesure. Il ne nous est pas encore possible malheureusement de prévoir quelle précision on peut espérer d'une telle détermination.

Signalons enfin qu'une difficulté supplémentaire vient compliquer l'optimisation : il existe un minimum minimorum trivial de la somme des carrés des écarts qui correspond au cas où la fonction $h$ se réduit à une impulsion de Dirac ; la déconvolution redonne alors le même résultat que le lissage, ce qui est rassurant... mais sans intérêt !

4. Résultats. - Nous donnerons deux exemples :

1. Un résultat positif : la figure 1 est relative à une portion de ligne de balayage, en émission secondaire.

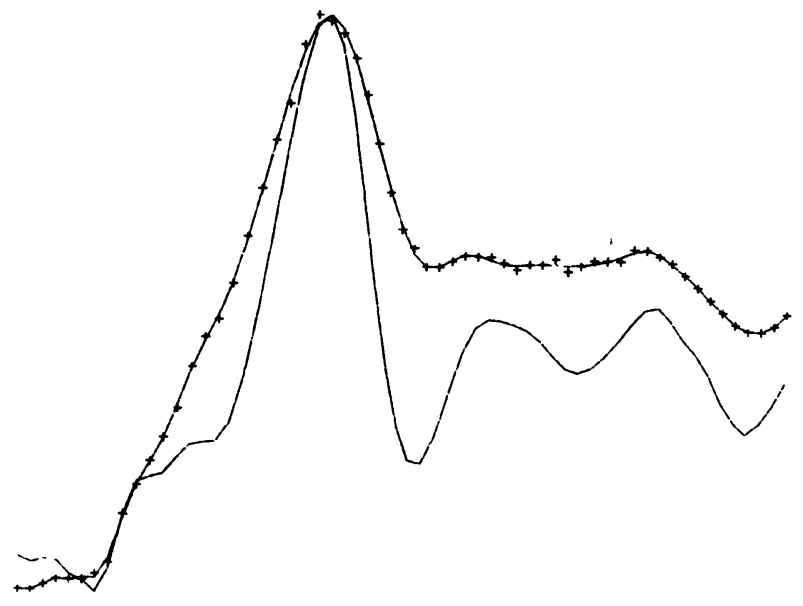

FIG. 1. -+++ points expérimentaux et courbe lissée. courbe déconvoluée.

Diverses fonctions $h$ simples (porte, gaussienne, fonction de Cauchy) n'ont donné aucun résultat; une somme pondérée de deux gaussiennes permet la déconvolution : « le pic principal » est beaucoup plus étroit, certains détails sont maintenant résolus. $\mathrm{La}$ figure 2 montre la ligne complète de balayage; la déconvolution est obtenue avec la même fonction $h$ que pour le début de la ligne. Ceci permet de penser que, si l'on voulait traiter plusieurs lignes d'une même image, voire l'image entière, l'optimisation ne serait à faire qu'une seule fois.

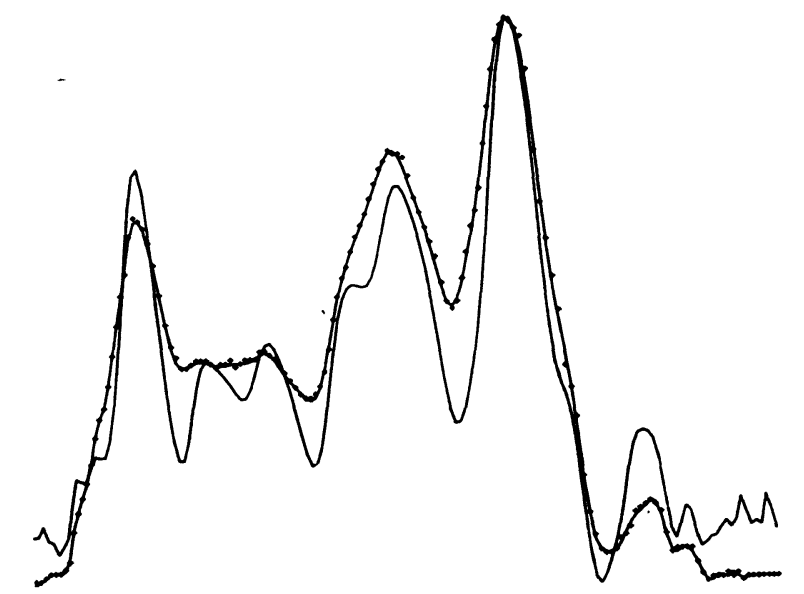

FIG. 2.

+++ points expérimentaux et courbe lissée. courbe déconvoluée.

- Un résultat négatif : pour obtenir un exemple avec les électrons rétrodiffusés seuls, nous avons choisi un eutectique orienté $\mathrm{Pb}-\mathrm{Sn}$ où l'on observe des précipités d'étain dans des lamelles de plomb [2]. Cet échantillon ne nous a pas permis d'obtenir de résultat acceptable. On peut penser que le plomb seul ou l'étain donneraient des fonctions d'échantillons très différentes; dès lors, il n'est pas possible de trouver une fonction commune pour un échantillon hétérogène.

5. Conclusion. - La déconvolution est opération délicate ; elle n'est pas toujours réalisable (matériaux hétérogènes). Effectuée sur une ligne de balayage seulement elle permet déjà d'améliorer la précision des mesures (par exemple mesure des longueurs de diffusion dans les semiconducteurs) et, en optimisant la fonction d'échantillon, d'obtenir des renseignements physiques sur le matériau étudié (distribution spatiale des électrons secondaires, des rétrodiffusés, pourcentage relatif des électrons émis). Lorsque l'on sera en mesure de traiter une image complète, on pourra améliorer considérablement la résolution du microscope à balayage ; il n'est pas exclu de pouvoir effectuer cette opération sur le microscope lui-même sans recours à un ordinateur.

Remerciements. - Nous remercions vivement M. B. Moraweck (Institut de Recherches sur la Catalyse) avec lequel nous avons eu de fructueuses discussions).

\section{Bibliographie}

[1] MAX, J., Méthodes et techniques de traitement du signal et applications aux mesures physiques (Masson et Cie, Paris) 1972, Tome I.

[2] Blanchin, M. G., Guinier, A., Petipas, C. et Sauvage, G.. Acta Metal. 20 (1972) 1251 